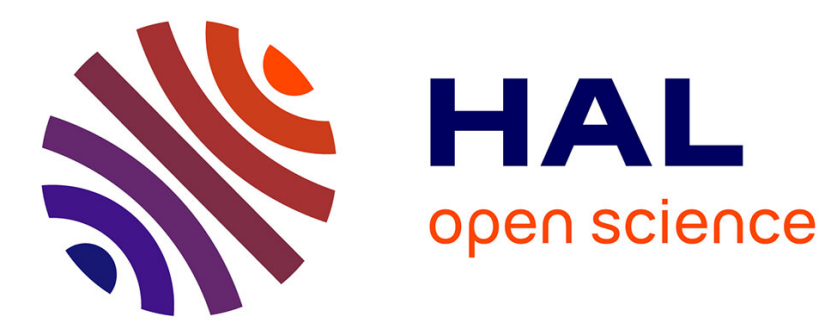

\title{
A modular bilateral haptic control framework for teleoperation of robots
}

\author{
Zeki Y. Bayraktaroglu, Omer F. Argin, Sinan Haliyo
}

\section{To cite this version:}

Zeki Y. Bayraktaroglu, Omer F. Argin, Sinan Haliyo. A modular bilateral haptic control framework for teleoperation of robots. Robotica, 2019, 37 (02), pp.338-357. 10.1017/S0263574718001042 . hal02017363

\section{HAL Id: hal-02017363 https://hal.sorbonne-universite.fr/hal-02017363}

Submitted on 5 Mar 2019

HAL is a multi-disciplinary open access archive for the deposit and dissemination of scientific research documents, whether they are published or not. The documents may come from teaching and research institutions in France or abroad, or from public or private research centers.
L'archive ouverte pluridisciplinaire HAL, est destinée au dépôt et à la diffusion de documents scientifiques de niveau recherche, publiés ou non, émanant des établissements d'enseignement et de recherche français ou étrangers, des laboratoires publics ou privés. 


\title{
A Modular Bilateral Haptic Control Framework for Teleoperation of Industrial Robots
}

\author{
Zeki Y. Bayraktaroglut*, Omer F. Arginł and Sinan Haliyo§ \\ †Istanbul Technical University, Mechanical Eng. Dept. \\ $¥$ Istanbul Technical University, Mechatronics Eng. Dept. \\ $\S$ SorbonneUniversite, Institut des Systemes Intelligents et de Robotique
}

\begin{abstract}
SUMMARY
This manuscript presents a novel approach to implement bilateral control loops between local haptic devices and remote industrial manipulators using a layer of simulation and virtual reality. The remote scene of manipulation has been visualized in an open-source software environment, where forward and inverse kinematics of the manipulators can be computed. Therefore, the explicit knowledge of mathematical models of the robots is not required for the implementation of the proposed bilateral control schemes. A haptic coupling has been designed between the human-operator and the task in the remote environment. Virtually introduced force feedback has contributed to the performance of the proposed bilateral loop by facilitating the adaptation of unexperienced humanoperators. Tele-manipulation with one remote manipulator has been experimentally demonstrated with the proposed controllers. Structural modularity of the bilateral haptic control schemes makes them directly extendable for the telemanipulation with multiple collaborative robots. Stability and transparency of the proposed bilateral haptic controllers have been theoretically and experimentally investigated.
\end{abstract}

KEYWORDS: Control of Robotic Systems; Haptic Interfaces; Man-Machine Systems; Teleoperation; Bilateral Control Systems.

\section{Introduction}

Theoretical aspects of bilateral haptic controllers have been rigorously investigated in literature. Many bilateral schemes with various assumptions of operating conditions and controllers have been proposed and analyzed. Nonlinearities in bilateral loops are generally introduced by the kinematics and dynamics of manipulators operating in remote environments. Linear techniques of stability analysis may apply in case the remote manipulator exhibits linear behavior. ${ }^{1,2}$ Stability analysis of bilateral teleoperation systems incorporating nonlinearities can be carried out in the general frame of the Lyapunov theory along with passivity approach.

Among earlier contributions, Anderson and Spong investigated the asymptotic stability and bilateral control of remote tele-operators. ${ }^{3,4}$ Niemeyer and Slotine published contributions to fundamental issues of teleoperation with studies of adaptive control and force reflection. ${ }^{5,6}$ Zhu and Salcudean, ${ }^{7}$ Hashemzadeh et al. ${ }^{8}$ and Sarras et al. ${ }^{9}$ proposed adaptive control techniques for teleoperation with time delays. Chopra et al. reported on tracking performance in bilateral teleoperation and problems related to communication networking.

Lee and Spong investigated passive bilateral teleoperation and presented an experimental setup with 2-DoF direct-drive robot. ${ }^{12}$ Nuño presented a teleoperation framework with an experimental setup including one haptic device and one remote

\footnotetext{
* Corresponding author. E-mail:zeki.bayraktaroglu@itu.edu.tr
} 
manipulator. ${ }^{13}$ Nuño et al. have shown that it is possible to control bilateral teleoperators with simple PD-like schemes with damping injection. ${ }^{14}$ The analysis has been validated by simulations. In, ${ }^{15}$ Nuño et al. proposed an experimental test-bed similar to that proposed in this work. The presented task consisted on moving the robot end-effector along a rail with a line restriction.

Desbats et al. established force-feedback teleoperation in nuclear spent fuel processing application, using an industrial robot as the remote manipulator. ${ }^{16}$ Soyguder and Abut proposed a modified wave variables method for the control of a single remote manipulator. ${ }^{17}$ The experimental work focused only on the motion control of the remote manipulator.

Stability issues of bilateral systems involving single remote manipulator have been clearly presented with analysis results. A firm theoretical base has now been established for the analysis of bilateral haptic systems. A number of computer simulations of proposed bilateral control systems have been reported. Most of the published research deal with the case of bilateral haptic interaction with virtual environments. However, practical implementations of teleoperation systems with physically real remote environments are rarely present in scientific literature or in industrial applications.

This paper proposes a novel modular framework to implement a bilateral haptic coupling between one haptic device and one robot manipulator operating in remote environments. The aim of the proposed design is to establish stable and robust telemanipulation framework with user-friendly and easy-to-use physical interfaces. Using an open source simulation software in the loop, the coupling has been achieved with the sole knowledge of kinematic parameters of the remote manipulator. Experimental results confirm the flexibility of the proposed control system structure which provides considerable ease in both implementation and remote operator handling.

In the bilateral control system proposed in this paper, haptic coupling is defined and computed in a layer of virtual reality environment, designed itself with an open source software interface. There are two major contributions implemented within this layer of simulation and virtual reality. First, the remote physical environment has been virtually reconstructed and simulated to provide the human-operator with a visual perception in real-time during tele-manipulation. The second contribution is about the virtual haptic coupling defined in the same simulation environment. Coupling forces computed in the virtual environment are being added to the feedback forces measured at the remote manipulation and the resulting total feedback force is being applied to the haptic device. Feedback of virtual coupling forces felt by the human-operator constitutes an additional mean of perception, contributing to a better overall perception of the remote environment.

Robot controller gains are usually tuned to behave best around some nominal motion dynamics, and there are always operational restrictions about allowable joint accelerations. Unexperienced human-operators are very likely to applying highly dynamic motion inputs to the remote environment side, which may result with poor tracking performance and instability of the bilateral loop. Feedback of the virtually introduced coupling forces to the human-operator makes him/her feel a resistance to the motion and helps improving the performance of the control loop by preventing him/her from applying high-frequency motion inputs.

The proposed haptic coupling design involving the virtual feedback forces introduces a low-pass filter behavior, filtering out high frequency motion inputs from unexperienced human-operators. The first contribution of this design to the performance of the teleoperation system is the imporvement of stability of the bilateral loop. Secondly, easy adaptation of unexperienced new users, thereby minimizing training periods is another contribution of the proposed methods.

In the following section, the bilateral haptic coupling used in the proposed control systems is presented. Motion control of remote manipulators as well as overall bilateral control schemes are presented in Section 3. Section 4 presents a Lyapunov-like analysis of the bilateral loop with a single remote manipulator, investigating in the stability 
conditions. Hardware and software used in implementations as well as the control parameters are presented in Section 5. Experimental results of tele-manipulation with single remote manipulator are given in Section 6. Section 7 presents conclusions.

\section{Bilateral haptic coupling with virtual spring-damper model}

Haptic coupling design between distant physical devices can be defined with respect to the behavior of the haptic device present in the bilateral loop. Two main design approaches are based on the impedance and admittance of the haptic device, also called as the master or local device.

In impedance approach, the master (local) device position is used as the motion reference for the slave (remote) manipulator. The slave-side force measurements are fed back to the haptic device, all with proper scaling factors. This kind of coupling is easily destabilized if different coefficients are used in force and motion scalings. Another limitation is that the user does not receive any information on the slave dynamics, except what is directly measured by a F/T sensor, usually mounted on the wrist of the remote manipulator.

In admittance approach, the master device is controlled as to mimic the slave manipulator dynamics. Forces applied by the user to the haptic device are transmitted as references for the force control of the slave manipulator, and its motion is fed back as the motion reference for the haptic device. This kind of coupling requires a F/T measurements at the master device. It should also be controlled in closed-loop to match slave device' $\mathrm{s}$ apparent mass, inertia and other mechanical behavior. This is considerably more complex, both for the hardware of the master device and identification of the slave manipulator.

As an overwhelming majority of the commercial haptic devices make only impedance available, where an indirect coupling mode through a virtual viscoelastic element is generally used. ${ }^{18}$ In this case, master and slave positions are considered each at opposite ends of a virtual spring-damper. Their relative motions deform this element, and the virtual force it produces, summed to the slave force sensor output, is used to forcecontrol the slave manipulator on one side and fed back to the user on the other. Tuning the stiffness and damping of this viscoelastic element allows for a compromise between the stability and transparency and is reported to give satisfactory results. This indirect coupling strategy is largely inspired by a large corpus of works on physicallybased simulations with haptic interaction. ${ }^{19,}{ }^{20}$ Several enhancements are studied, such as automatic tuning of spring-damper parameters, or adaptative schemes. ${ }^{21}$

There is however an important difference in implementation with respect to the nature of the remote environment. In simulation applications where the remote scene is virtual, the haptic coupling is established between the master device and manipulated (virtual) object. In tele-operation applications where real physical interactions are controlled in remote environments, the coupling is defined between the master (haptic) device and the end-effector of the slave (remote) manipulator. Hence, the user feedback stems from the slave manipulator, but not the manipulated load. Moreover, force control of the slave side is often not straightforward and requires the knowledge and use of a dynamic model. Generally only a single slave manipulator can be remotely operated without an elaborate scheme.

The framework presented here aims to establish haptic coupling in task space, between the master device and manipulated object (load) directly. The load may be handled by one or several collaborating remote manipulator(s). Consequently, dynamics of the remote manipulator is neglected in servo-control and the slave device is reduced to a simple motion transmitter, constituting a stand-alone component of the bilateral loop. In this modular approach, any robot whose basic kinematic parameters are available can serve as the remote manipulator.

The proposed framework is based on two simultaneous parallel couplings. A virtual reality and physical simulation environment is used as middle layer, and includes the manipulated object, and the remote manipulator. It provides the real-time simulation of 
dynamics, i.e. object motion is calculated from a set of forces, and inverse kinematics of the slave robot, considering the load as the goal position. Both those features are easily available, as open-source libraries or commercial products.

The first coupling, borrowing the conventional haptic physical simulation technique, is established between the haptic interface and virtual object using a spring-damper model. This generates a force to move the virtual object and gives haptic feedback about the object' s simulated inertia to the user. Collisions or haptic guides can be easily added. The second coupling is between the end-effector of the remote manipulator handling the real object, and the virtual object. This link is unilateral: the spring-damper force is only fed to the virtual object to reflects any position error in the real remote-side. The remote manipulator is controlled in joint space, with the configuration of the virtual slave robot as its motion references. Hence, this second coupling generates an information on the dynamics of the remote manipulator. This information can be augmented by a F/T sensor on the slave-side.

Several contributions stems from this double coupling through the layer of simulation and virtual reality. The remote physical environment has been virtually reconstructed and simulated to provide the human-operator with a visual perception in real-time during tele-manipulation. If collision detection is provided by the simulator, it is very straightforward to implement safe zones, haptic guides, or other augmented visual or audio feedback.

From a perception perspective, the user receives a feedback related to both object' $\mathrm{s}$ and the remote manipulators' motion and inertia. The ratio between those two can be easily tuned through homothetic coefficients on both couplings.

In the bilateral schemes proposed in this work, control of the remote manipulator is based on the sole knowledge of the basic kinematics, i.e. link lengths and joint definitions. The basic bilateral control scheme can be easily extended to the case of collaborating remote manipulators: a large load to be manipulated at several points will have different handling points, each representing the goal of IK problem of one slave manipulator. Hence the user does not need to care about the trajectories of each manipulator, but only the load itself. He will be however constrained in his motion by the physical interaction and object dynamics through kinesthetic feedback. Human motor context is expected to deal with this situation quite well.

\subsection{Virtual spring-damper model and total feedback force}

The position references generated by the human-operator through the haptic interface, after scaling, are denoted by ${ }_{T}$ in task frame. A virtual spring-damper model is designed $\underline{X}^{r}$

to achieve the transmission of the motion references to the robot controller as shown in Figure 1. Haptic coupling forces are assumed to result from the interaction with this virtual spring-damper interface.

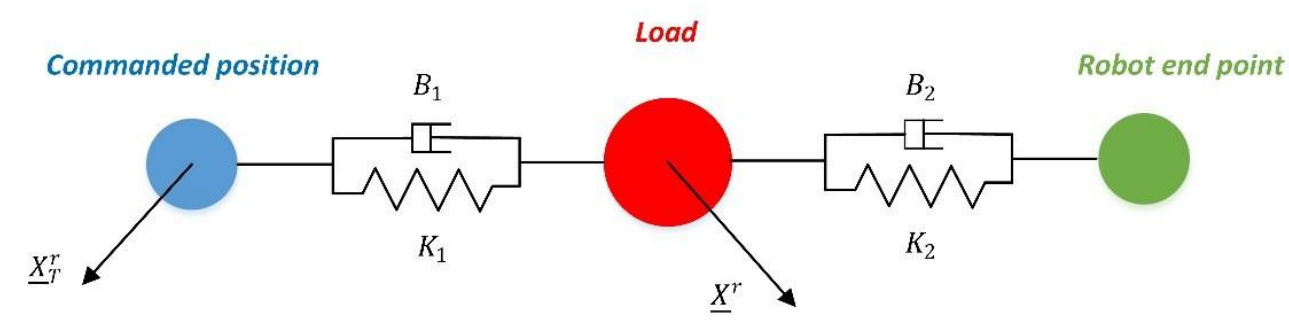

Fig. 1. Virtual spring-damper model.

Dynamics introduced in the haptic loop is then given by the following equation:

$$
m \underline{\underline{X}}+B_{2} \underline{\dot{X}}^{r}+K_{2} \underline{X}^{r}=B_{1} \underline{X}_{T}^{r}+K_{1} \underline{X}_{T}^{r}
$$


The resulting haptic coupling force $f_{C}$ and the total force $f_{T F}$ to be applied to the haptic interface are written as follows:

$$
\begin{gathered}
\left.f_{C}=K_{1} \underline{X}_{r}-\underline{X}^{r}\right)+B_{1} \underline{X}_{T}^{r}-\underline{X}^{r} \\
f_{T F}=f_{C}+f_{T}
\end{gathered}
$$

\begin{tabular}{|c|c|}
\hline$K_{x}, K_{f}$ & $\begin{array}{l}\text { Diagonal position and force scaling matrices for the output motion } \\
\text { of the haptic interface and for the input force to the haptic interface }\end{array}$ \\
\hline$K_{i}, B_{i}$ & Diagonal stiffness and damping matrices of the virtual spring model \\
\hline & Mass of the virtual spring-damper model \\
\hline$m_{l}$ & Mass of the manipulated object \\
\hline$\underline{X}_{H}$ & $\begin{array}{l}\text { Cartesian coordinates commanded by the haptic interface / in Haptic } \\
\text { frame }\end{array}$ \\
\hline$\underline{V}_{H}$ & Velocity vector of the haptic interface / in Haptic frame \\
\hline$\underline{\bar{X}}_{H S}$ & $\begin{array}{l}\text { Scaled coordinates commanded by the haptic interface / in Haptic } \\
\text { frame }\end{array}$ \\
\hline$\underline{X}_{T}^{r}$ & $\begin{array}{l}\text { Cartesian coordinates commanded by the haptic interface / in Task } \\
\text { frame }\end{array}$ \\
\hline$\underline{X}^{r}$ & Reference Cartesian coordinates / in Task frame \\
\hline$\underline{X}^{T}$ & Calculated end-effector position / in Task frame \\
\hline $\bar{V}$ & Velocity vector of the manipulator end-effector / in Task frame \\
\hline$\overline{\bar{q}}^{r}$ & Reference and Measured joint positions vector / in Joint frame \\
\hline$\underline{e}_{q}$ & Joint position errors vector / in Joint frame \\
\hline$\underline{e}_{f}$ & Force errors vector / in Task frame \\
\hline$\vec{\tau}$ & Joint torque vector / in Joint frame \\
\hline$f_{e}$ & External interaction forces vector / in Task frame \\
\hline$f_{T}^{e}$ & Measured end-effector force vector/in Task frame \\
\hline$f_{C}$ & Coupling force vector / in Task frame \\
\hline$f_{T F}$ & Feedback force vector / in Task frame \\
\hline$f_{H}^{T} f_{H S}$ & Measured and Scaled end-effector force vectors / in Haptic frame \\
\hline$S_{u}, S_{e}$ & Stiffness' of the human operator and environment sides \\
\hline$Z_{u}, Z_{e}$ & Impedances of the human operator and environment sides \\
\hline
\end{tabular}

\section{Nomenclature}

\subsection{Bilateral haptic control system}

The bilateral haptic control system for teleoperation proposed in this work can be presented as given in Figure 2. In this scheme, a human-operator controls the motion of a remote robot through the proposed haptic coupling for manipulation purposes. Three main blocks representative of static and dynamic subsystems can be identified in this bilateral loop.

Scaling of motion and force signals are linear operations and establish static relationships between the operator-side and task environment-side variables.

The Blender, ${ }^{22}$ an open source software used as the main component of the bilateral loop, executes three tasks. First, the kinematic chain and parameters of the robot manipulator are defined in Blender' s virtual environment, and the remote environment is visualized for the human-operator. Second, the dynamic behavior of the virtual springdamper model is simulated in Blender. Third, Blender computes the forward and inverse kinematics of the manipulator. Forward and inverse coordinate transformations define static mappings between joint and task space of the manipulator. Performance of these 


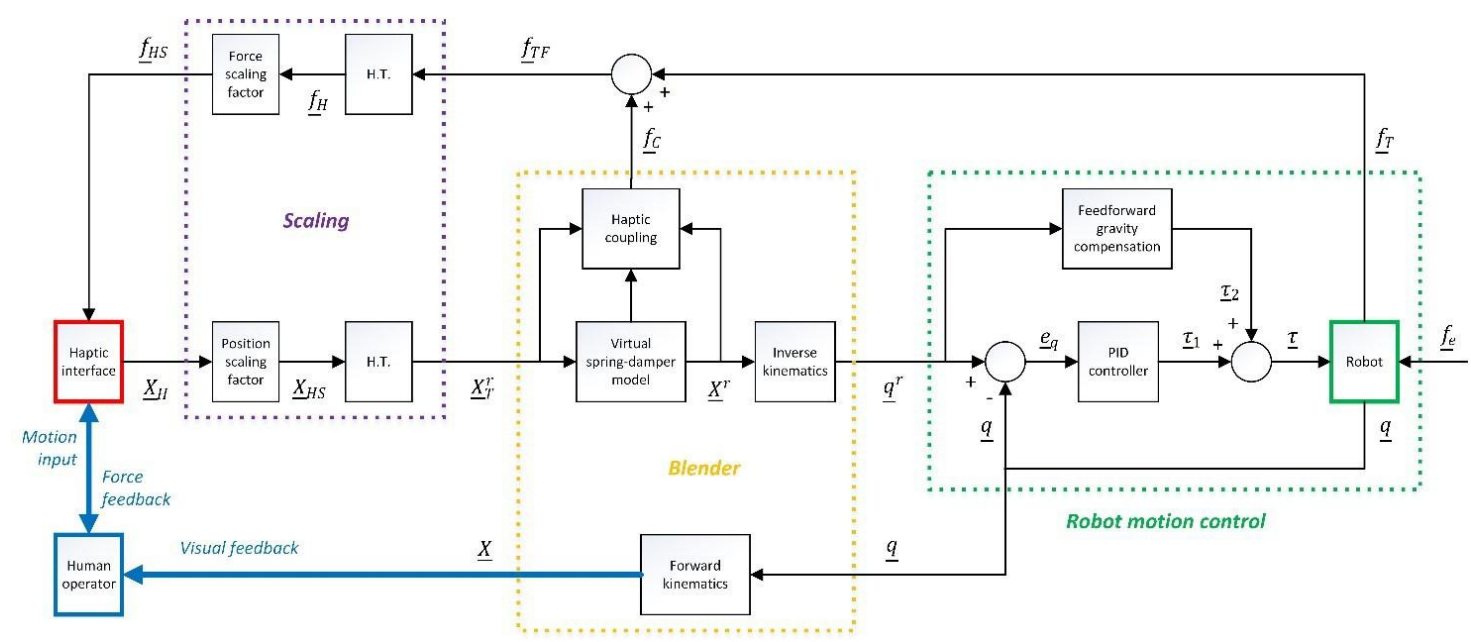

Fig. 2. Bilateral haptic coupling between one haptic device and one remote manipulator.

computational tasks by an extendable software environment contribute greatly to the compactness and simplicity of the proposed bilateral loop.

The remote robot, supposed to manipulate objects and interact with its environment is controlled in a feedback + feedforward loop with decentralized PIDs for each DoF. Dynamics of this subsystem can be modeled by nonlinear equations of motion. Time delays in the communication channels are assumed to be constant.

\subsection{Position and force scaling}

Workspaces of the haptic interfaces are generally reduced with respect to those of industrial manipulators. Manipulation/interaction tasks may require larger workspaces than those of haptic devices. Therefore, the motion input provided by the haptic interface has to be amplified to be compatible with the physical task defined in the manipulator' $\mathrm{s}$ workspace. Position scaling factor for the output motion of the haptic interface will be denoted by the (3 3) diagonal matrix $K_{x}$.

Amplitudes of interaction forces of the manipulators may depend on parameters related to the task to be achieved, such as the mass of an object to be manipulated. Amplitudes of continuous forces in haptic interfaces are generally less than $10 \mathrm{~N}$. Therefore, the interaction forces measured with 6-axis F/T transducers have to be scaled before being fed back to the haptic interfaces. Force scaling factor for the output motion of the haptic interface will be denoted by the (3 3) diagonal matrix $K_{f}$.

The use of diagonal matrices to set the position and force scaling factors allows the implementation of individually weighed scaling factors along the axis' of the world frame.

\subsection{Forward and inverse coordinate transformations}

Motion input provided by the human operator through the haptic interface is generated in task space and transmitted toward the environment through the virtual spring-damper model presented in Section 2.2. $\underline{X}_{T}$ is the $(3 \times 1)$ position vector representing the position only of the task space target point. The task space vector is then extended to the ( $\& 1)$ position and orientation vector $\underline{X}^{r}$ such that the gripper remains is a constant orientation in task space.

The robot motion controller is itself implemented in joint space. Therefore, the task space references $\underline{X}^{r}$ have to be transformed into joint space references $\underline{q}^{r}$. The inverse kinematics of the manipulator is achieved in virtual (Blender) environment. 


\section{Robot control}

In this work, the experimental setup of the proposed teleoperation system has been established. The fundamental bilateral scheme (Figure 2) describes the manipulation system with one haptic device and one remote manipulator.

In the haptic coupling presented in Section 2, one 6-axis industrial manipulator is used to manipulate objects and interact with the environment. An independent joint control scheme with decentralized PIDs for the motion control of the manipulator is proposed.

Independent joint control schemes result with the simplest closed-loop structures possible for the motion control of manipulator arms. Although the dynamics of the remote maipulator is neglected, implementation of independent joint controllers can be shown to be satisfactory in many applications with relatively slow and smooth motions. In the haptic coupling presented in Figure 2, the default workspace of the robot is reduced and reference velocities and accelerations are provided by the human operator. The control signal is strengthened with the addition of a feedforward action for gravity compensation.

The robot control loop shown in Figure 2 is based on independent joint controller with feedforward gravity compensation. The PID control law in joint space is given as follows:

$$
\underline{\tau}_{1}(t)=K_{p} \quad \underline{e}_{q}(t)+{\frac{1}{T_{i}}}^{r} \underline{e}_{q}(\tau) d \tau+T_{d} \frac{d \underline{e}_{q}(t)}{d t}
$$

where $\underline{e}_{q}=\underline{q}^{r}-q$ is the joint position error, and $K_{p}, T_{i}$ and $T_{d}$ denote $(6 \times 6)$ diagonal matrices for the PID parameters.

The feedforward action compensating for the external torque $\underline{\tau}_{2}$ due to gravitational acceleration is calculated as a nonlinear function of the robot configuration, and the total input torque applied to the robot joints is given by:

$$
\underline{\tau}=\underline{\boldsymbol{\tau}}_{1}+\underline{\boldsymbol{\tau}}_{2}
$$

The robot equations of motion can be written in standard form as follows:

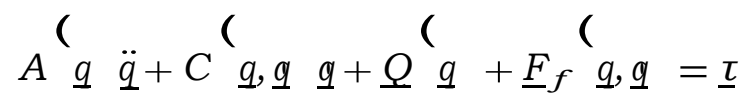

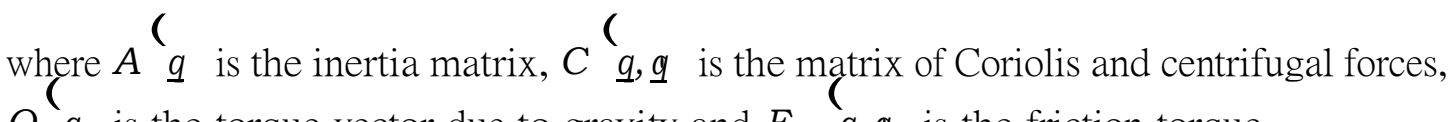
$Q \underline{q}$ is the torque vector due to gravity and $\underline{F}_{f} \underline{q}, \underline{q}$ is the friction torque.

Lyapunov stability of the closed-loop dynamics given by Eqs. (5) and (6) has been proved with the following Lyapunov function:

$$
V=\frac{1}{2} \cdot q^{T} A q+\frac{1}{2} \cdot \underline{e}_{q}^{T} K_{p} e_{q}
$$

Application of the invariant set theorem has shown the asymptotic stability of the closed-loop dynamics as given in refs. [23, 24].

\section{Stability and transparency of the bilateral haptic loop}

\subsection{Stability}

Stability of bilateral teleoperation has been investigated by Nuño et al. in refs. [13-15, $25,26]$ and by Hua in ref. [27]. In ref. [13], a general theoretical framework allowing the stability analysis of various control schemes present in the literature is proposed. The framework is based on the Lyapunov stability theory with passivity-based formulations. A comparative analysis of performances of different controllers shows that bilateral loops with PID type controllers are robust to variable time-delays and exhibit stable bilateral coupling. Time-delays may have important influence on the loop stability. Stability of 
bilateral loops with constant and variable time-delays has been investigated as well in refs. $[13,25-27]$. Stability analysis presented in this section follows a similar approach as given in the above mentioned publications. The haptic coupling between one haptic device and a single remote manipulator as presented in Fig. 2 is considered in the following analysis.

4.1.1. Properties and assumptions. Based on the equations of motion robot manipulators as given in Eq. (6), closed-loop dynamics of the bilateral haptic control system proposed in this work can be represented as follows:

$$
\begin{aligned}
& A_{m} \stackrel{(}{q}_{m} \ddot{q}_{m}+C_{m} \stackrel{(}{q}_{m}, \underline{q}_{m} \underline{q}_{m}=\underline{\tau}_{m}-\underline{\tau}_{u s e r} \\
& A_{b} \stackrel{(}{q}_{b} \ddot{q}_{b}+C_{b} \stackrel{(q}{b}_{b}, \underline{q}_{b} \underline{q}_{b}=\underline{\tau}_{b} \\
& A_{s} \underline{q}_{s} \ddot{q}_{s}+C_{s} \underline{q}_{s}, \underline{q}_{s} \underline{q}_{s}+\underline{Q}_{s} \stackrel{q}{q}_{s}=\underline{\tau}_{s}+f_{e}
\end{aligned}
$$

In above equations (8)-(10), indices $m, b$ and $s$ denote respectively the master haptic

device, virtual Blender model and slave (remote) manipulator. $f_{e}$ is the vector of interaction force between the remote manipulator and environment, and $\underline{\tau}$ user is the force/torque applied by the user through the haptic interface.

In the bilateral loop proposed in this work, communication delays between are assumed to be constant valued and defined as follows:

$T_{1}$ : time-delay between the haptic device and blender model;

$T_{2}$ : time-delay between the blender model and remote manipulator;

- $T_{3}$ : time-delay between the haptic device and remote manipulator.

Control inputs to be applied to the master and slave manipulators are given as follows:

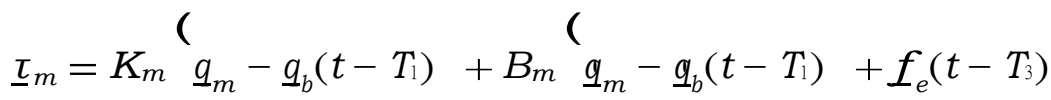

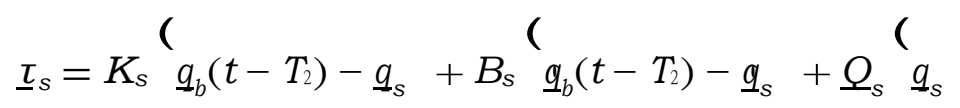

Assumptions on the parameters of robot manipulators are represented by the following mathematical properties:

- (P1) $A q$ is a symmetric positive definite inertia matrix and there are positive constants $r$ and $r$ such that $r I \geq A \underline{q} \geq r \underline{b}$

- (P2) For all $q_{i}, x, y \in R^{n x^{1}}$, there are positive scalars $p_{i}$ such that $p_{i}|x| \mathbf{I} \mid y \mathbf{|} \geq$ $\mathbf{I} C_{i}\left(q_{i}, x\right) y \mathbf{I}$

- (P3) There are positive scalars $\beta_{i}$ such that $U_{i}\left(q_{i}\right) \geq \beta_{i}$ where $U_{i}\left(q_{i}\right)$ represents the potential energy of the robot, satisfying $g_{i}\left(q_{i}\right)=\partial U_{i}\left(q_{i}\right) / \partial q_{i}$.

- (P4) $A_{i} \underline{q}_{i}=C_{i} \underline{q}_{i}+C_{i} \underline{q}_{i}$

Lemma 1 (ref. [27]). For a positive-definite matrix $\Upsilon$, the following inequality holds:

$$
2 a^{T}(t){ }_{t-T}^{r_{t}} b(\sigma) d \sigma-{ }_{t-T}^{r_{t}} b^{T}(\sigma) \Upsilon b(\sigma) d \sigma \leq \bar{T} a^{T}(t) \Upsilon{ }^{-1} a(t)
$$

where $a(t)$ and $b(t)$ are vector functions and $T$ is a time-varying scalar satisfying $0 \leq$ $T \leq \bar{T}$. 
Passivity of the human operator and environment can be represented by the following inequalities:

$$
-{ }_{0}^{q_{m}^{t}}{ }_{m}^{T}(\sigma) \tau_{\text {user }}(\sigma) d \sigma \geq 0 \quad, \quad-{ }_{0}^{t_{-} q_{s}{ }^{T}}(\sigma) f_{e}(\sigma) d \sigma \geq 0
$$

4.1.2. Lyapunov stability. If the system given by Eqs. (8)-(10) is controlled through the inputs proposed in Eqs. (11)-(12) with the following bounds on $\tau_{b}$ and $\underline{\tau} u_{u s e r}$ :

$$
\underline{\tau}_{b} \geq K_{m}\left(\underline{q}_{m}-\underline{q}_{s}\right) \quad, \quad \underline{\tau}_{u s e r} \geq \frac{1}{2} f_{e}\left(t-T_{3}\right)
$$

then $q_{i}$ and $q_{m}-q_{s}$ are shown to be bounded with the following Lyapunov function candidtate:

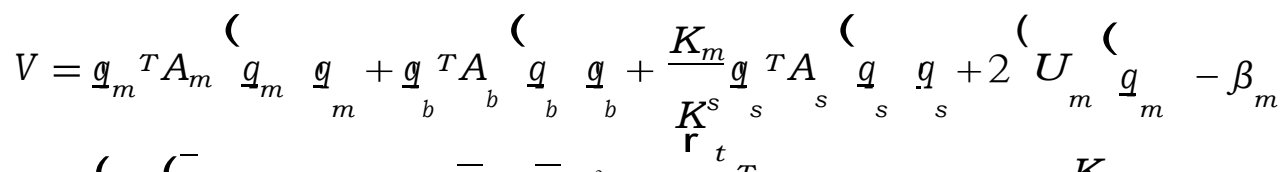

$$
\begin{aligned}
& +2{ }^{(} U_{s}\left({ }_{q}^{-}-\beta_{s}+K_{m}\left(\bar{q}_{b}-\bar{q}_{m}\right)^{2}-2{ }^{t_{T}}{ }_{\left(q_{m}\right.}(\sigma) \bar{\tau}_{u s e r}(\sigma)+\frac{K_{m}}{K_{s}} d_{s}(\sigma) f(\sigma)\right) d \sigma
\end{aligned}
$$

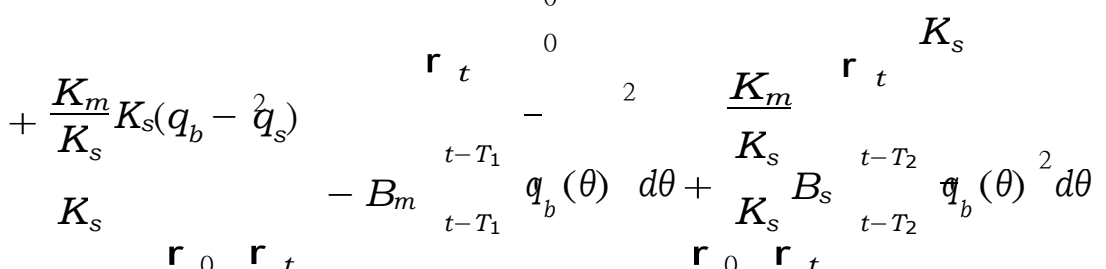

$$
\begin{aligned}
& +_{-T_{1} \quad t+\theta} \underline{q}_{b}^{T}(\sigma) S \underline{q}_{b}(\sigma) d \sigma d \theta+{ }_{-T_{2}} \quad t+\theta_{b}{ }^{T}(\sigma) Z q_{b}(\sigma) d \sigma d \theta
\end{aligned}
$$

where $S$ and $Z$ are positive definite symmetric matrices.

Proof of the Lyapunov stability is given in Appendix.

4.1.3. Asymptotic stability. Since the proposed bilateral loop is a time-varying system, in order to prove the asymptotic stability of the bilateral loop in presence of negative semidefinite only $V$, one has to show the uniform continuity of $V$. According to Barbalat' $s$ Lemma, ${ }^{28}$ uniform continuity of $V$ implies the convergence $V \rightarrow 0$ as $t \rightarrow \infty$.

In order to prove the uniform continuity of $V$, its time-derivative must be shown to be bounded:

$$
\begin{aligned}
& \ddot{V}=2 \ddot{q}_{m}{ }^{T}{ }^{(} \bar{T}_{1} K_{m}{ }^{2} S^{-^{1}}+B_{m} \quad q_{m}+2 \ddot{q}_{b}^{T}{ }^{(} T_{1} S+\bar{T}_{2} Z+\frac{K_{m}}{K_{s}} B_{s}-B_{m} \quad q_{b} \\
& +2 \ddot{q}_{s}{ }^{T}\left(\bar{T}_{2} K_{m}{ }^{2} Z^{-1}-\frac{K_{m}}{K_{s}} B_{s} \underline{q}_{s}+2 \ddot{q}_{m}{ }^{T}\left(f\left(t-T_{3}\right)-2 \underline{\tau}_{\text {user }}\right)\right. \\
& +2 \underline{q}_{m}^{T}\left(\left(1-T_{3} \underline{f}_{e}\left(t-T_{3}\right)-2 \underline{\tau}_{u s e r}\right)+2 \ddot{q}_{b}^{T}\left(K_{m}\left(q_{m}-q_{s}+\underline{\tau}_{b}\right)\right.\right. \\
& +2 q_{b}^{T}\left(K_{m} \underline{q}_{m}-\underline{q}_{s}+\underline{\boldsymbol{\tau}}_{b}\right)
\end{aligned}
$$

Based on closed-loop dynamics equations (8) and (10), and control laws (11) and (12), joint accelerations of the master and slave manipulators are given as follows:

$$
\begin{aligned}
\ddot{q}_{m}=A_{m^{-1}}\left(K_{m} \underline{q}_{m}-\underline{q}_{b}\left(t-T_{1}\right)+\left(B_{m}\right.\right. & \left.\left.-C_{m}\right) \underline{q}{ }^{m}\right) \\
& +A_{m^{-1}}-B_{m} \underline{q}_{b}\left(t-T_{1}\right)+\underset{e}{f\left(t-T_{3}\right)}
\end{aligned}
$$




$$
\begin{aligned}
& \ddot{q}=A_{b}{ }^{-1}-C_{b} \cdot \underline{q}_{b}+\underline{\tau}_{b} \\
& \ddot{q}_{s}=A_{s}{ }^{-1}{ }_{K_{s}}\left(\underline{q}_{b}\left(t-T_{2}\right)-\underline{q}_{s}+A_{s}{ }^{-1}{ }_{B_{s}}\left(\underline{q} \gamma_{s} t-T_{2}\right)-\underline{q}_{s}-C_{s} \underline{q}{ }_{s}\right.
\end{aligned}
$$

Boundedness of $\underline{q}_{i}, \underline{q}_{i}, f_{e}$ and $\underline{\boldsymbol{\tau}}_{b}$ in Eqs. (18) and (20) along with (P1) imply that of $\ddot{q}_{i}$. The first 3 terms of $V$ are therefore bounded. Boundedness of the remaining 4 terms of $\ddot{V}$ is guaranteed according to conditions given in Eqs. (14) and (15).

Therefore, according to Barbalat' $\mathrm{s}$ lemma, the proposed bilateral haptic system is shown to be asymptotically stable.

\subsection{Transparency}

Proceeding as in refs. [1] and [2], the definition of the transparency of the haptic coupling is based on impedances or stiffness at the human operator and environment sides, as proposed in refs. [29] and [30]. Perfect transparency in a bilateral haptic loop is assumed to be achieved when both side impedances (or stiffness' ) are equal. Impedances and stiffness felt by the human operator $\left(Z_{u}\right.$ and $S_{u}$ ) and of the environment ( $Z_{e}$ and $S_{e}$ ) are defined as follows:

$$
Z_{u}=\frac{f_{H S}}{\mid \underline{V_{H} \mid}} \quad, \quad S_{u}=\frac{f_{H S}}{\left|X_{H}\right|} \quad, \quad Z_{e}=\frac{f_{T F}}{\mid \underline{V} \mathbf{I}} \quad S_{e}=\frac{f_{T F}}{|\underline{X}|}
$$

Impedances and stiffness' have been calculated according to definitions (21), based on force and motion measurements.

\section{Implementation}

\subsection{Hardware and software}

The haptic interface of the proposed bilateral coupling is a Phantom Premium 1.5 High Force device. The haptic coupling has been implemented with 6-axis Stäubli Rx160 manipulator arm (Figure 3). The manipulator is equipped with 6-axis ATI F/T transducers and single-DoF on/off gripper. The robot motion control is implemented through the Low Level Interface (LLI) provided in ref. [31]. Sampling frequencies of the robot controller and the haptic interface are $250 \mathrm{~Hz}$ and $1 \mathrm{kHz}$ respectively.

The dynamic behavior of the virtual spring model proposed in section II as well as the inverse coordinate transformations are handled by an open source 3D creation software. ${ }^{22}$ Once the kinematic chains of the manipulator arms are defined in the graphical interface of the Blender software, forward and inverse kinematics are computed by appropriate solvers (Figure 4).

The Blender is also provided with a physics engine, with which the dynamics of the manipulation and physical interactions can be simulated. Masses manipulated in experiments have been defined in Blender interface (Figure 5).

The purpose of the use of the virtual environment in the proposed bilateral coupling system lies in the simplicity and modularity of loop design. Kinematic parameters of the manipulator arm as well as the parameters of the virtual spring model can be easily modified through the Blender graphical interface. In case the manipulator is replaced by another one, the use of the virtual environment offers a user-friendly redesign of the bilateral loop.

\subsection{Control parameters}

Parameters of the bilateral loop belong to three sub-system shown in Figure 2: Scaling, Blender and Robot motion control. 


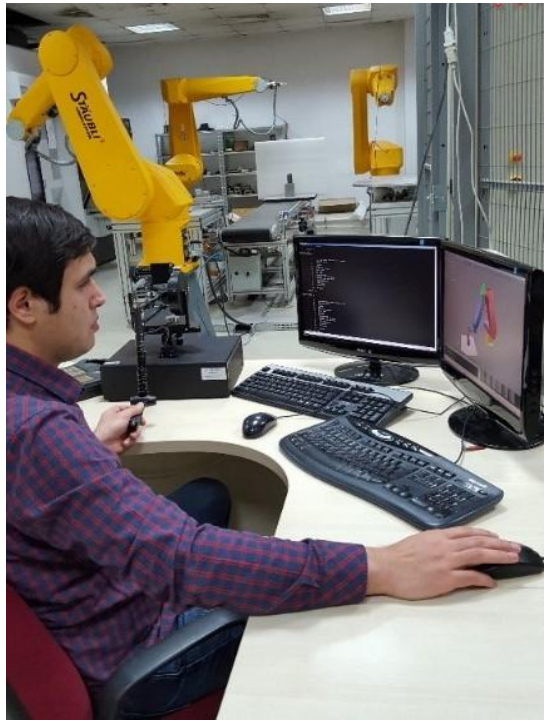

Fig. 3. Remote manipulators.

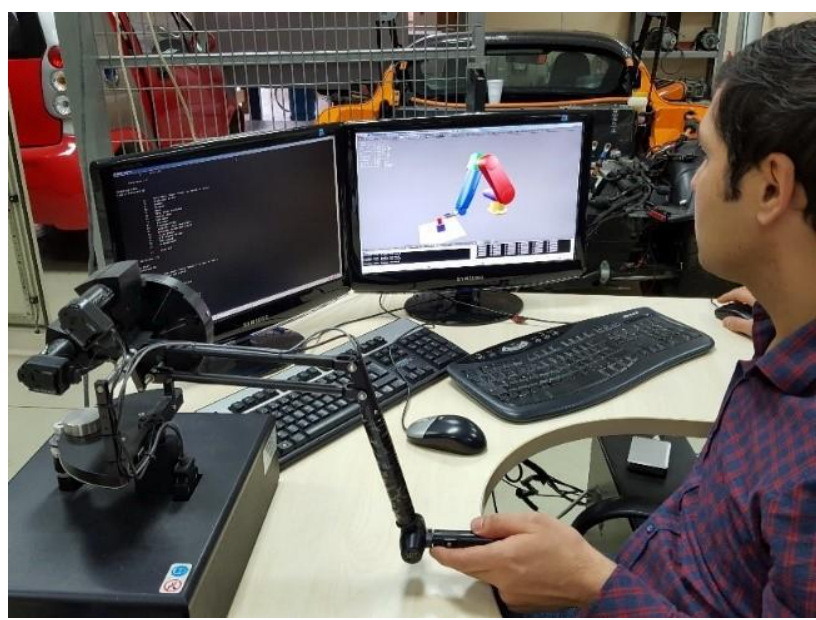

Fig. 4. Haptic device and virtual Blender interface.
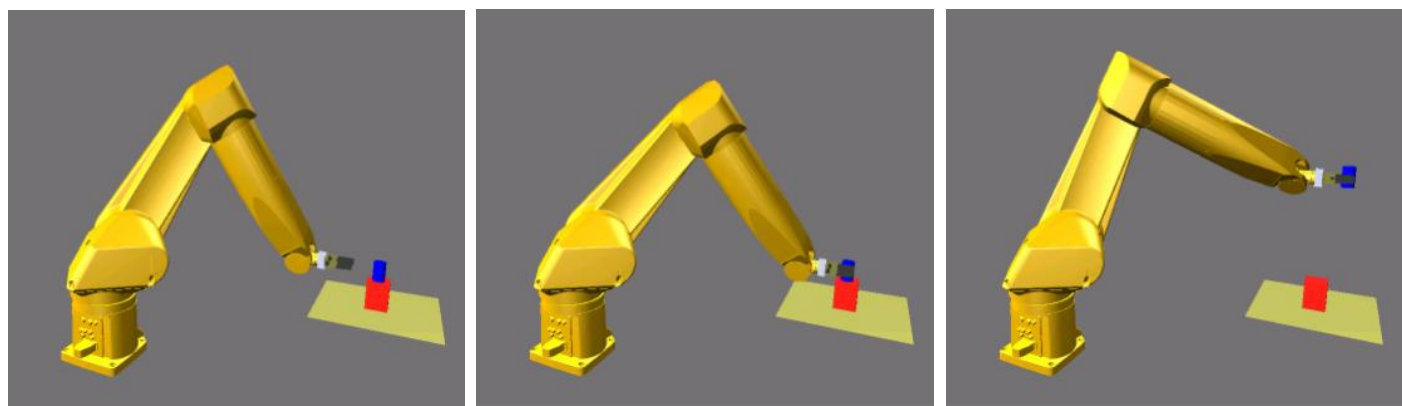

Fig. 5. Remote manipulator in the virtual (Blender) environment. a) Approach phase, b) Handling of the object, c) Manipulation.

5.2.1. Scaling factors. The position and force scaling factors $K_{x}=\frac{X H S}{\underline{X}_{H}}$ and $K_{f}=\frac{f}{H S}$ are determined with respect to the constraints related to the haptic device. The workspace of the haptic device is smaller than the robot' $\mathrm{s}$ workspace and the sub-(work)space in 
which the robot operates depends on the task. The position scaling is then chosen so as to establish a convenient correspondence between these workspaces.

Choice of the force scaling depends primarily on the maximum force available on the haptic device. The maximum force of the haptic device has a limited value with respect to interaction forces that may occur in the physical environment. Accuracy of the F/T transducer being variable over a range of measured amplitudes, it displays decisive effects on the quality of force feedback. Experiments have been planned such that the interaction forces in the remote environment remain close to the linear range of the F/T transducer. The force scaling is then chosen to obtain realistic force feedback without violating the capacity of the haptic device.

5.2.2. Virtual spring-damper and robot controller parameters. Stiffness and damping parameters of the virtual spring model presented in section II have been determined for desired damping ratio $\zeta_{d}$. Damping ratios satisfying $0,7 \zeta_{c<} 1$ have been implemented in experiments. Parameters have been selected such that the resulting natural frequency $\omega_{n}$ remain far from the structural frequencies of the haptic device. Note that stiffer springs will contribute to better position tracking but will result with higher coupling forces, which will in turn induce oscillations in system signals.

Finally, the PID parameters $K_{p}, T_{i}$, and $T_{d}$ of the robot controller given in Eq. (4) have been experimentally tuned.

\section{Experiments}

In the experiments, haptic manipulation of rigid objects has been achieved with one remote manipulator and one remote manipulators. The bilateral control loop applied in experiments is the one given in Figure 2. The manipulated object has mass $m_{l}=2 \mathrm{~kg}$ and scaling factors have been implemented as follows: $K_{x}=30, K_{f}=$

Parameters of the virtual spring-damper model have been implemented as follows:

$m=1, B_{2}=35, K_{2}=600$. Note that these values of the virtual model parameters result with a damping ratio of $\zeta=0,714$ and natural frequency $\omega_{n}=24,5 \mathrm{rad} . \mathrm{s}^{-1}$.
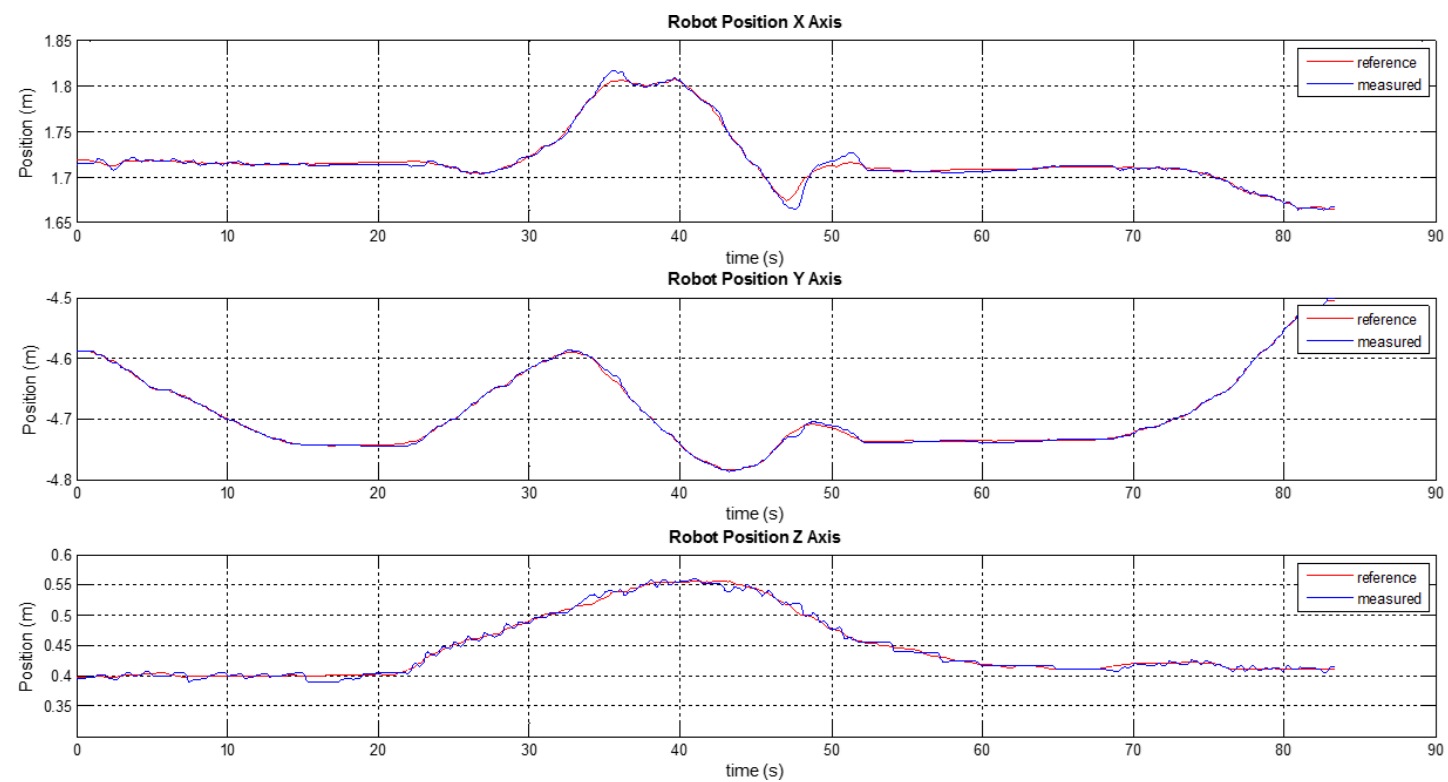

Fig. 6. Remote manipulator position tracking in task space.

Tracking performance of the remote manipulator in task space is depicted in Figure 6. The average position tracking error is $3,46 \mathrm{~mm}$. Measured and scaled end-effector forces of the remote manipulator are given in Figure 7. 

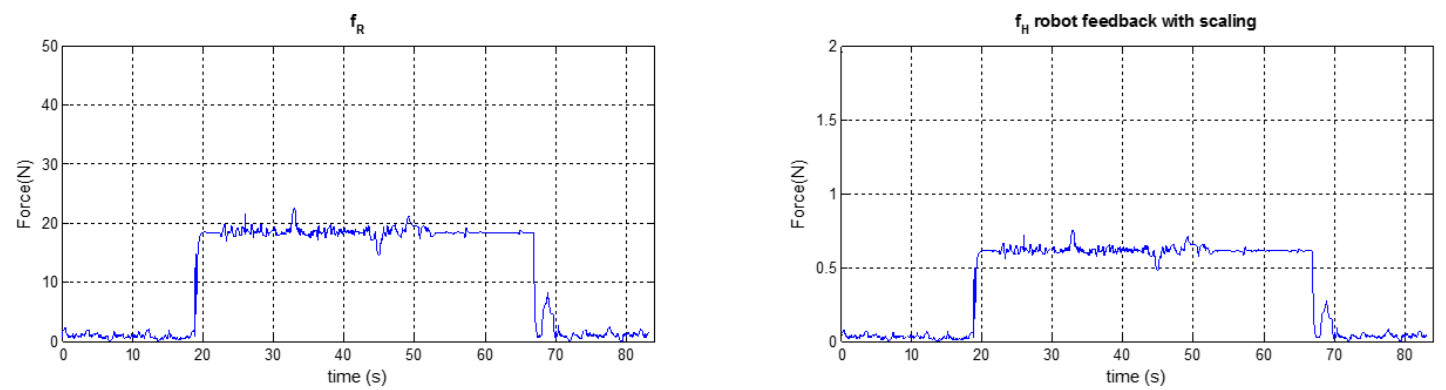

Fig. 7. Remote manipulator (a) measured and (b) scaled end-effector forces.

The virtual spring force given in Figure 8(a) is artificially introduced to the control loop and provides the user with an apparent inertia feeling while commanding the remote manipulator. Figure 8(b) depicts the total haptic feedback force applied to the haptic device interface, with the contributions of forces due to the haptic coupling and manipulated load.
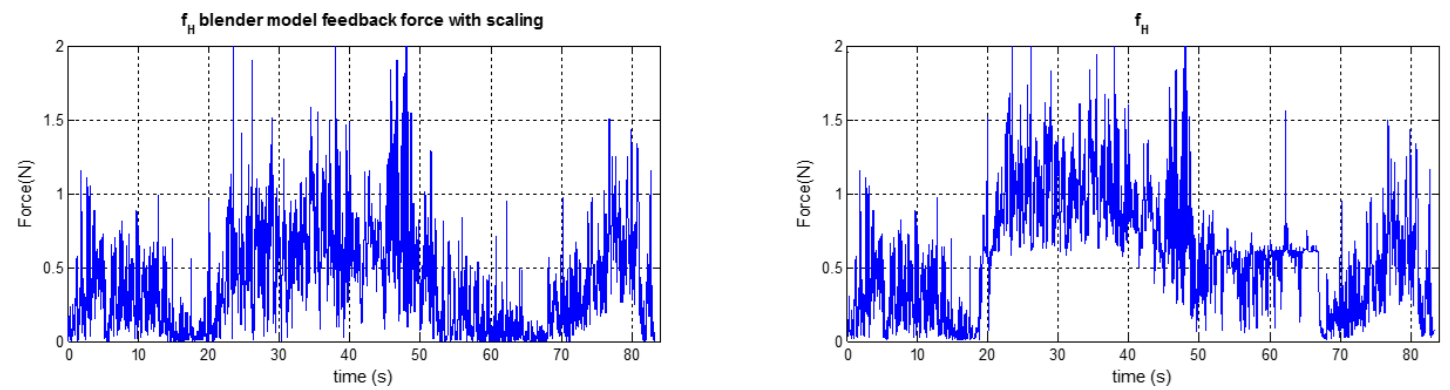

Fig. 8. (a) Virtual spring force, (b) Total haptic feedback force.

Figures 9 and 10 show the stiffness' and impedances calculated for the manipulator end-effector and haptic device. Since the implemented scaling factors satisfy $K_{x} \cdot K_{f}=1$, the same order of magnitudes as well as trends for stiffness' and impedances can be observed at both ends of the bilateral loop as expected.
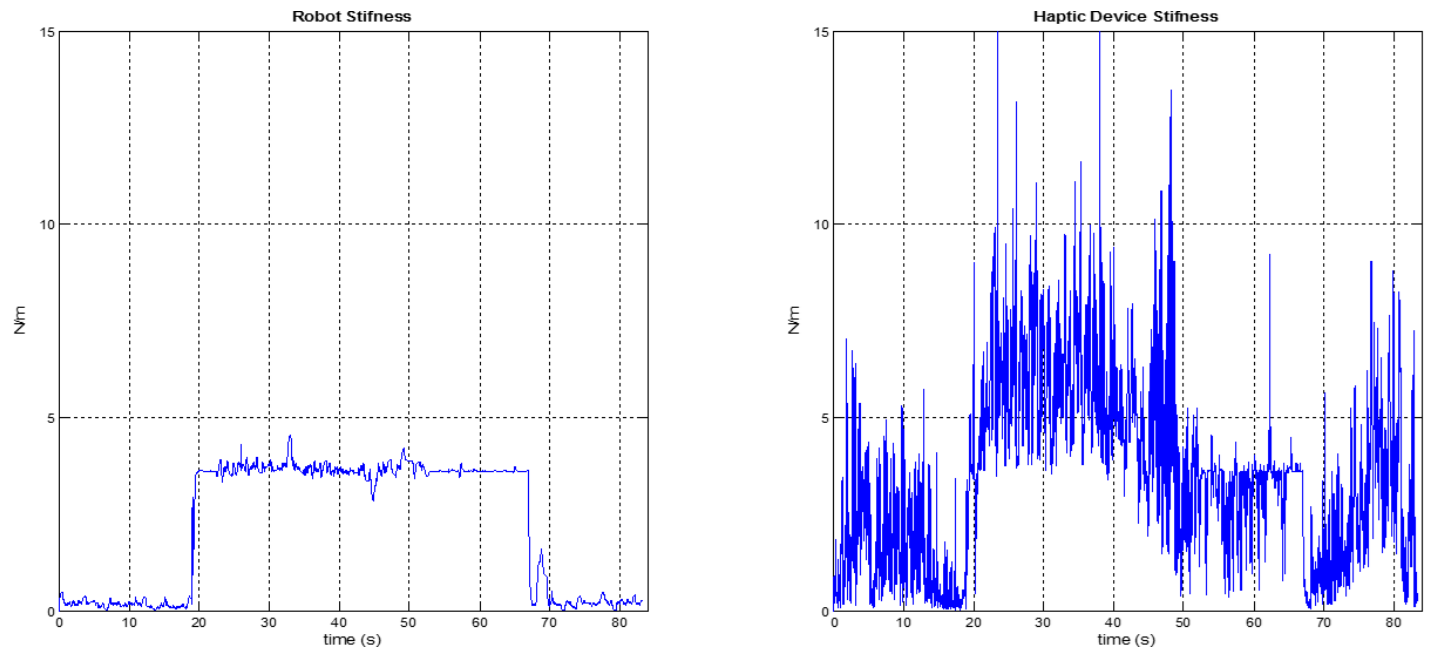

Fig. 9. (a) Remote manipulator and (b) Haptic device stiffness'. 

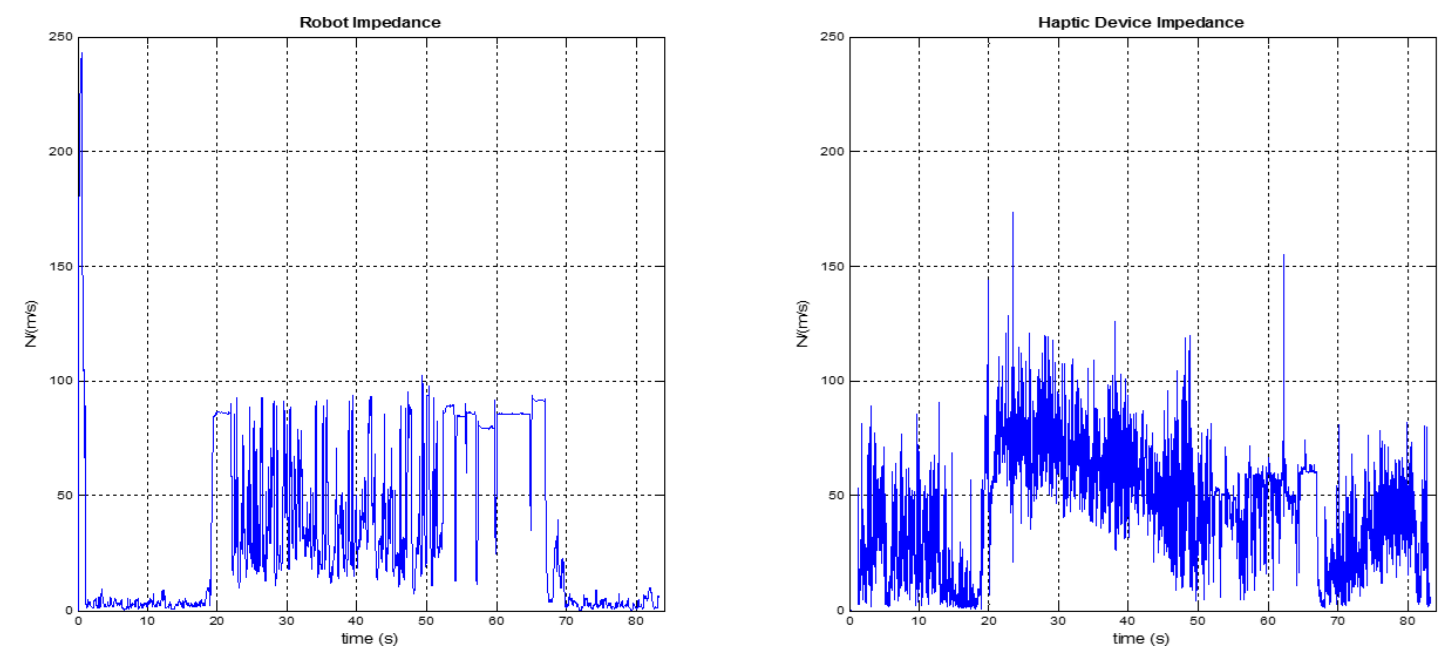

Fig. 10. (a) Remote manipulator and (b) Haptic device impedances.

Experimental results presented in this section have been obtained with an experienced user. The proposed bilateral control system has been experimented by unexperienced users as well. At the experiments conducted with untrained users, similar behaviors have been observed with respect to the fundamental performance criteria such as stability, transparency and position tracking in task space. Figure 11 presents the tracking error performances with one experienced (reference) user and 6 other unexperienced users.

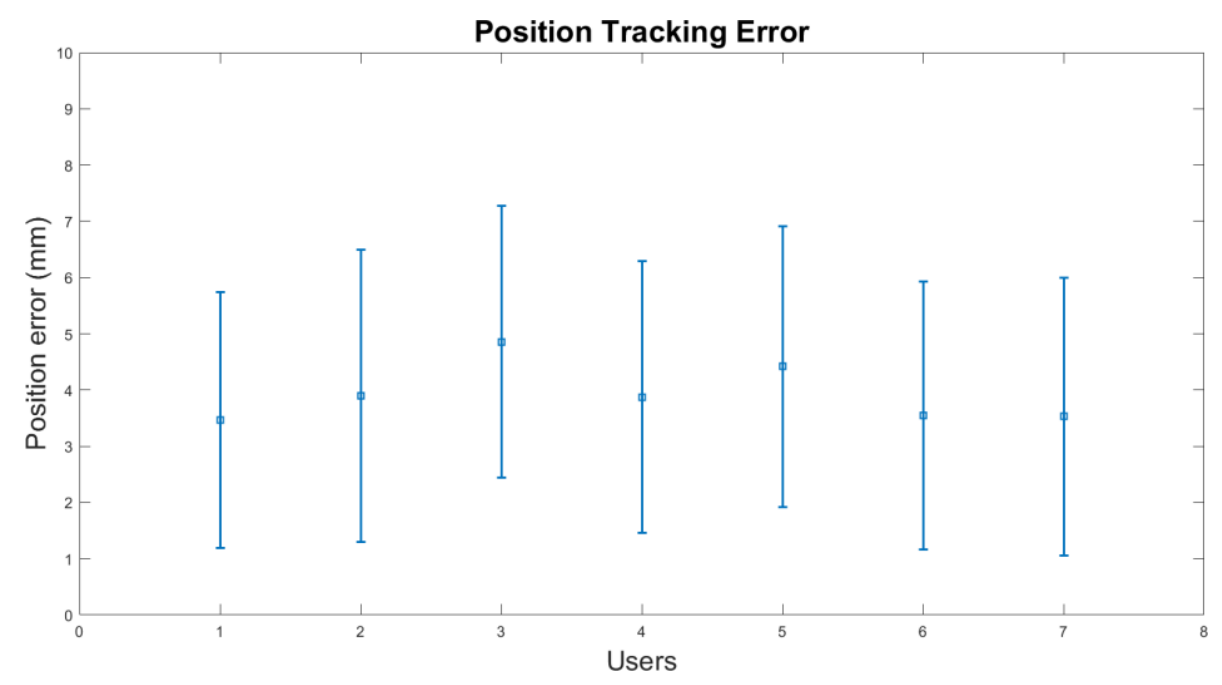

Fig. 11. Position tracking errors with one trained (1st user) and 6 untrained users.

\section{Conclusion}

A bilateral haptic control loop between one haptic device and one remote manipulator has been proposed in this paper.

Asymptotic stability conditions for the bilateral loop with position controlled single remote manipulator has been theoretically investigated. Stable operations of the proposed bilateral haptic loop have been experimentally achieved by trained and untrained users. Transparency of the proposed bilateral haptic loops have been experimentally evaluated as presented in section 6 . 
Implementation and experimental validation of the proposed controller have been achieved in a modular programming environment. Note that the proposed bilateral control scheme can be easily extendable to the collaboration tasks with multiple remote manipulators.

Definition of the kinematic chain involving geometric parameters of the manipulator is sufficient for the implementation of the proposed bilateral controllers. Since no further mathematical model is needed, any industrial robot arm can be easily integrated as one remote manipulator.

In position control of industrial manipulators, motion references with excessive accelerations may result with unstable behavior of the system and the actuator currents are usually limited for safety reasons. In the absence of any haptic interface, motion references from the human-operator side may exceed the allowable input frequencies. Then proper operation of the bilateral control system would require training periods of the users. The haptic coupling proposed in this paper provides the user with a feeling of apparent inertia while commanding the remote manipulator through the haptic device. This artificially introduced 2nd order dynamics filters out the high frequency motion references from the user and therefore allows unexperienced users to easily achieve telemanipulation.

\section{References}

1. A. Bolopion, B. Cagneau, D. Haliyo and S. Régnier, "Analysis of stability and transparency for nanoscale force feedback in bilateral coupling," J. of Micro-Nano Mechatronics 4(4), 145-158 (2008).

2. A. Mohand Ousaid, A. Bolopion, S. Haliyo, S. Régnier and V. Hayward, "Stability and transparency analysis of a teleoperation chain for microscale interaction," In: Proceedings of the IEEE International Conference on Robotics and Automation (2014) pp. 5946-5951.

3. R.J. Anderson and M.W. Spong, "Asymptotic stability for force reflecting teleoperators with time delay," In: Proceedings of the IEEE International Conference on Robotics and Automation (1989) pp. $1618-1625$.

4. R.J. Anderson and M.W. Spong, "Bilateral control of teleoperators with time delay," IEEE Trans. on Automatic Control 34(5), 494-501 (1989).

5. G. Niemeyer and J.J.E. Slotine, "Stable adaptive teleoperation," IEEE J. of Oceanic Eng. 16(1), 152162 (1991).

6. G. Niemeyer and J.J.E. Slotine, "Transient shaping in force reflecting teleoperation," In: Proceedings of the International Conference on Advanced Robotics (1991) pp. 261-266.

7. W.H. Zhu and S.E. Salcudean, "Teleoperation with adaptive motion/force control," In: Proceedings of the IEEE International Conference on Robotics and Automation (1999) pp. 231-237.

8. F. Hashemzadeh, T. Hassanzadeh, M. Tavakoli and G. Alizadeh, "Adaptive control for state synchronization of nonlinear haptic telerobotic systems with asymmetric varying time delays," $J$. of Intelligent and Robotic Systems 68(3), 245-259 (2012).

9. I. Sarras, E. Nuño and L. Basañez, "An adaptive controller for nonlinear teleoperators with variable time-delays," J. of the Franklin Institute 351(10), 4817-4837 (2014).

10. N. Chopra, M.W. Spong, R. Ortega and N.E. Barabanov, "On tracking performance in bilateral teleoperation," IEEE Trans. on Robotics 22(4), 861-866 (2006).

11. N. Chopra, P. Berestesky and M.W. Spong, "Bilateral teleoperation over unreliable communication networks," IEEE Trans. on Control Systems Technology 16(2), 304-313 (2008).

12. D. Lee and M.W. Spong, "Passive bilateral teleoperation with constant time delay," IEEE Trans. on Robotics 22(2), 269-281 (2006).

13. E. Nuño, "Haptic guidance with force feedback to assist teleoperation systems via high speed networks," In: Proceedings of the IEEE International Symposium on Robotics(2006) pp. 1-14.

14. E. Nuño, R. Ortega, N. Barabanov and L. Basañez, "A globally stable PD controller for bilateral teleoperators," IEEE Trans. on Robotics 24(3), 753-758 (2008).

15. E. Nuño, L. Basañez and R. Ortega, "Passivity-based control for bilateral teleoperation: A tutorial," Automatica 47, 485-495 (2011).

16. P. Desbats, F. Geffard, G. Piolain and A. Coudray, "Force-feedback teleoperation of an industrial robot in a nuclear spent fuel reprocessing plant," Industrial Robot 33(3), 178-186 (2006).

17. S. Soyguder and T. Abut, "Haptic industrial robot control with variable time delayed bilateral teleoperation," Industrial Robot 43(4), 390-402 (2016).

18. J.E. Colgate, M.C. Stanley and J.M Brown, "Issues in the haptic display of tool use," In: Proceedings of the IEEE/RSJ International Conference on Intelligent Robots and Systems (1995) pp. 140-145.

19. C.B. Zilles and J.K. Salisbury, "A constraint-based god-object method for haptic display," In: Proceedings of the IEEE/RSJ International Conference on Intelligent Robots and Systems (1995) pp. $146-151$. 
20. B.M. Howard and J.M. Vance, "Desktop haptic virtual assembly using physically based modelling," Virtual Reality 11(4), 207-215 (2007).

21. X. Hou and O. Sourina, "Stable Adaptive Algorithm for Six Degrees-of-Freedom Haptic Rendering in a Dynamic Environment," Vis. Comput. 29(10), 1063-1075 (2013).

22. Blender, "https://www.blender.org/" (2017).

23. S. Arimoto and F. Miyazaki, "Stability and robustness of PID feedback control for robot manipulators of sensory capability," In: Proceedings of the International Symposium on Robotics Research (1984) pp. 783-799.

24. W. Khalil and E. Dombre, "Modeling, identification and control of robots," Kogan Page Science (2004).

25. E. Nuño, L. Basañez and M. Prada, "Asymptotic stability of teleoperators with variable time-Delays," In: Proceedings of the IEEE International Conference on Robotics and Automation (2009) pp. 43324337.

26. E. Nuño, L. Basañez, R. Ortega and M.W. Spong, "Position tracking for non-linear teleoperators with variable time Delay," Int. J. of Robotics Research 28(7), 895-910 (2009).

27. C.C. Hua and X.P. Liu, "Delay-dependent stability criteria of teleoperation systems with asymmetric time-varying delays," IEEE Trans. on Robotics 26(5), 895-910 (2010).

28. J.J.E. Slotine and W. Li, "Applied nonlinear control," Prentice Hall (1991).

29. D. Lawrence, "Stability and transparency in bilateral teleoperation," IEEE Trans. on Robotics and Automation 9(5), 624-637 (1993).

30. P.F. Hokayem and M.W. Spong, "Bilateral teleoperation: An historical survey," Automatica 42(12), 2035-2057 (2006).

31. Stäubli Manual, “Arm - RX series 160 family Instruction Manual” (2008).

\section{Appendix: Proof of the Lyapunov stability}

The first time derivative of the Lyapunov function candidate with (P3) and (P4) can be written as follows:

$$
\begin{aligned}
& V=\underline{q}_{m}{ }^{T}{ }^{(} C_{m}+C_{m}{ }^{T} \underline{q}_{m}+2 \underline{q}_{m}{ }^{T}{ }^{(} \underline{\boldsymbol{\tau}}_{m}-\underline{\boldsymbol{\tau}}_{u s e r}-C_{m} \underline{q}_{m}+\underline{q}_{b}{ }^{T}{ }^{(} C_{b}+C_{b}{ }^{T} \underline{q}_{b} \\
& +2 \underline{q}_{b}{ }^{T} \underline{\tau}_{b}-C_{b} \underline{q}_{b}+\frac{K_{m}}{K_{s}} q_{s}{ }^{T}{ }_{C}+C_{s}{ }_{s} \underline{q}+2 \frac{K_{m}}{q^{s}} \underline{s}_{s}{ }^{(} \underline{\tau}_{s}+\underline{F}_{e}-C_{s} \underline{q} \\
& -2 K_{m} \mathfrak{q}_{m}^{T} \underline{q}_{m}-\underline{q}_{b}+2 K_{m} \mathfrak{q}_{b}^{T} \underline{q}_{m}-\underline{q}_{b}\left(+2 K_{m} \mathfrak{q}_{b}^{T} \underline{q}_{b}-\underline{q}_{s}\right.
\end{aligned}
$$

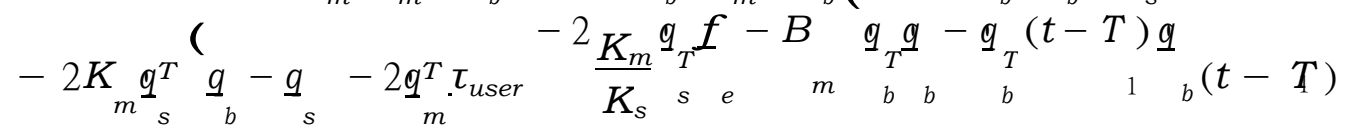

$$
\begin{aligned}
& +\frac{K_{m}}{K_{s}} B_{s}\left(\mathfrak{q}_{b}^{T} q_{b}-q_{b}^{T}\left(t-T_{2}\right) \underline{q}_{b}\left(t-T_{2}\right)+\bar{T}_{1} \mathfrak{q}_{b}^{T} S q_{b}\right. \\
& -{ }_{t-T_{1}}^{r^{s}} q_{b}^{T}(\sigma) S q_{b}(\sigma) d \sigma+\bar{T}_{2} q_{b}^{T} Z q_{b}-{ }_{t-T_{2}}^{r_{-} q_{b}^{T}}(\sigma) Z q_{b}(\sigma) d \sigma
\end{aligned}
$$

(A1) can be rearranged as follows:

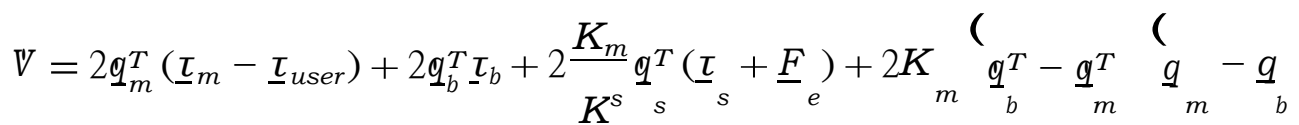

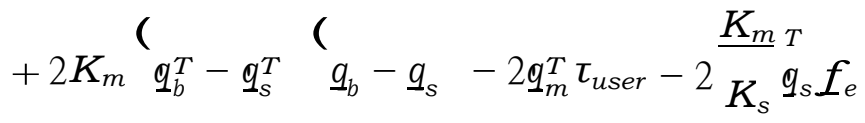

$$
\begin{aligned}
& -B_{m}{ }^{(} \mathfrak{q}_{b}^{T} q_{b}-\mathfrak{q}_{b}^{T}\left(t-T_{1}\right) \mathfrak{q}_{b}\left(t-T_{1}\right)+\frac{K_{m}}{K_{s}} B_{s} \underset{s}{(} \mathfrak{q}_{b}^{T} \underline{q}_{b}-\mathfrak{q}_{b}^{T}\left(t-T_{2}\right) \mathfrak{q}_{b}(t-\underline{T}) \\
& +\bar{T}_{\Gamma} \mathbb{q}_{b}^{T} S q_{b} \quad{ }_{t-T_{1}}^{r_{t}-\bar{q}_{b}^{T}}(\sigma) S q_{b}(\sigma) d \sigma+\bar{T}_{2} \mathfrak{q}_{b}^{T} Z q_{b}-{ }_{t-T_{2}}^{t}-\bar{q}_{b}^{T}(\sigma) \overline{Z_{q_{b}}}(\sigma) d \sigma
\end{aligned}
$$


Substituting the above given expressions and bounds of $\underline{\tau}_{m}, \underline{\tau}_{b}$ and $\underline{\tau}_{s}$ :

$$
\begin{aligned}
& V=2 K_{m} \mathfrak{q}_{m}^{T}\left(\underline{q}_{m}-\underline{q}_{b}\left(t-T_{1}\right)+2 B_{m} \mathfrak{q}_{m}^{T} \underline{q}_{m}-\underline{q}_{b}\left(t-T_{1}\right)+2 \mathfrak{q}_{m}^{T} f\left(t-T_{3}\right)\right. \\
& -2 \mathfrak{q}_{m}^{T} \underline{\underline{\tau}} u s e r+2 \mathfrak{q}_{b} \underline{\boldsymbol{\tau}}_{b}+2 \boldsymbol{K}_{m} \mathfrak{q}_{s}^{T} \underline{q}_{b}\left(t-T_{2}\right)-\underline{q}_{s}+2 \frac{K_{m}}{K_{s}} B_{s} \mathfrak{q}_{s}^{T}\left(\mathfrak{q}_{b}\left(t-T_{2}\right)-\underline{q}_{s}\right.
\end{aligned}
$$

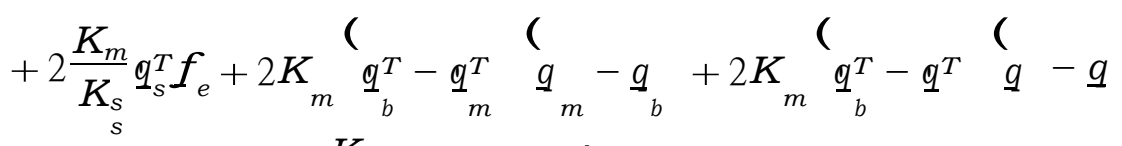

$$
\begin{aligned}
& -2 \mathfrak{q}_{m}^{T} \underline{\underline{\tau}}_{u s e r}-2 \frac{K_{m}}{K_{s}} \mathfrak{q}_{s} f_{e}-B m \underset{b}{(} \mathfrak{q}_{b}^{T} \mathfrak{q}_{b}-\underset{b}{\mathfrak{q}_{T}}\left(t-T_{1}\right) \underset{b}{(t-T)}(t) \\
& +\frac{K_{m}}{K_{s}} B_{s} \underset{b}{\left(\mathfrak{q}_{b}^{T} \mathfrak{q}\right.}-\mathfrak{q}_{b}\left(t-T_{2}\right) \underset{b}{q}(t-\underset{2}{T})+\bar{T}_{1} \mathfrak{q}_{b}^{T} S \underline{q}_{b} \\
& -{ }_{t-T_{1}}^{\mathbf{r}_{t_{-}}} \boldsymbol{q}_{b}(\sigma) S \boldsymbol{q}_{b}(\sigma) d \sigma+\bar{T}_{2} \boldsymbol{q}_{b} Z \boldsymbol{q}_{b}^{T}-{ }_{t_{-T}}^{\mathbf{r}_{t_{2}}} \\
& t-T_{1} \\
& { }_{t-T_{2}}^{t-T_{2}} q_{b}(\sigma) Z q_{b}(\sigma) d \sigma
\end{aligned}
$$

Manipulating (A3) by adding and substracting $\underline{q}_{b}$ into the $1^{\text {st }}$ and $6^{\text {th }}$ terms, it can be rearranged as follows:

$$
\begin{aligned}
& V=2 K_{m} \underline{q}_{m}^{T}\left(\underline{q}_{b}-\underline{q}_{b}\left(t-T_{1}\right)+2 B_{m} \underline{q}_{m}^{T} \underline{q}_{m}-\underline{q}_{b}\left(t-T_{1}\right)+2 \mathfrak{q}_{m}^{T} f_{e}\left(t-T_{3}\right)\right.
\end{aligned}
$$

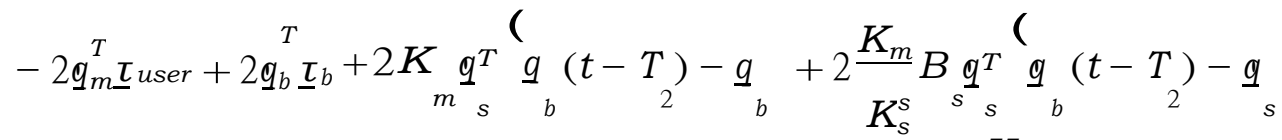

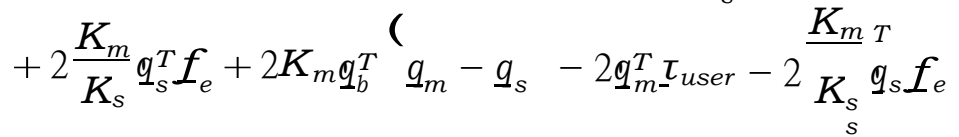

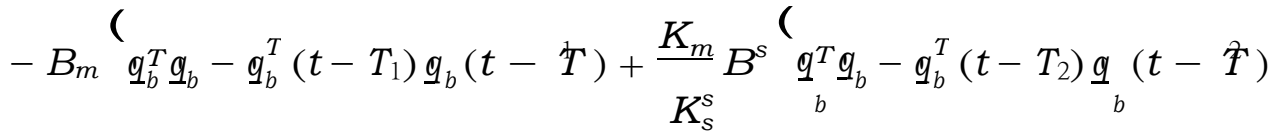

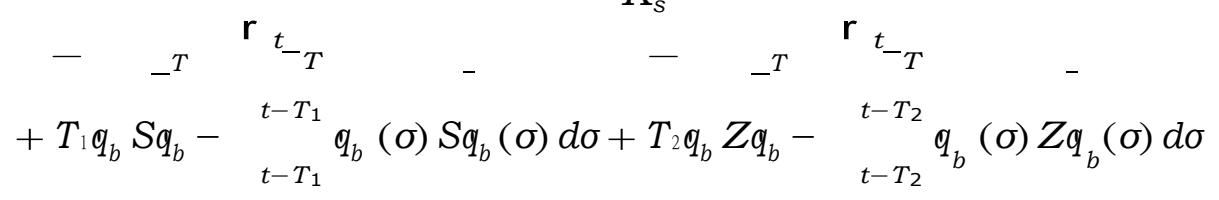

Taking into account the following equality:

$$
\bar{q}_{i}(t-T)-\bar{q}_{i}=\mathrm{r}_{T_{-}}
$$

and expanding the $2^{\text {nd }}$ and $7^{\text {th }}$ terms:

$$
q_{i}(t \quad \sigma) d \sigma
$$

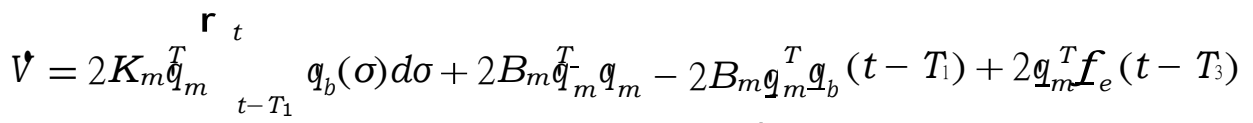

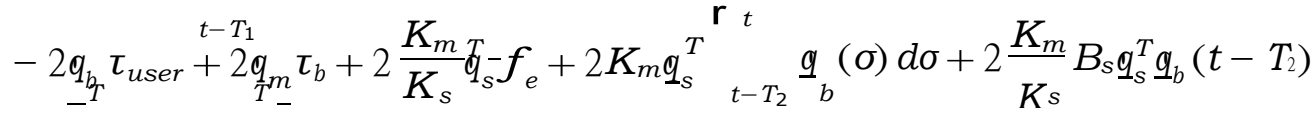

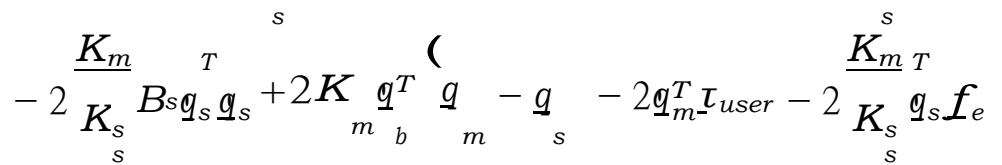

$$
\begin{aligned}
& -B_{m}\left(\mathfrak{q}_{b}^{T} \mathfrak{q}_{b}-\mathfrak{q}_{b}^{T}\left(t-T_{1}\right) \underline{q}_{b}(t-T)+\frac{K_{m}}{s} B^{s} \mathfrak{q}^{T} \mathfrak{q}_{b}-\mathfrak{q}_{b}^{T}\left(t-T_{2}\right) \underline{q}(t-\mathfrak{T})\right. \\
& +T_{1} q_{b} S q_{b}-\quad q_{b}(\sigma) S q_{b}(\sigma) d \sigma+T_{2} q_{b} Z q_{b}-
\end{aligned}
$$


18

$$
\begin{array}{ll}
-\quad-{ }^{T} & \mathrm{r}_{t_{-T}} \\
& \\
& t-T_{1} \\
& t-T_{1}
\end{array}
$$

Haptic teleoperation of industrial robots $K_{s}$

$\begin{array}{cc}{ }_{-}^{b} & r_{t_{-T}} \\ & t-T_{2} \\ & t-T_{2}\end{array}$


Applying the following bound:

$$
2 q_{\frac{q}{i j}}(t-T) \leq q_{i}^{T} q_{i}+q_{j}^{T}(t-T) q_{j}(t-T)
$$

to the $3^{r d}$ and $9^{\text {th }}$ terms of (A5):

$$
\begin{aligned}
& V=2 K_{m} \stackrel{\underline{q}}{m}_{m}^{r_{t}}{ }_{t-T_{1}}{ }_{\boldsymbol{q}}(\sigma) d \sigma+2 B_{m} \underline{q}_{m}^{T} \underline{q}_{m}-B_{m} \underline{q}_{m}^{T} \underline{q}_{m}-B_{m} \underline{q}_{b}^{T}\left(t-T_{1}\right) q_{b}\left(t-T_{1}\right)
\end{aligned}
$$

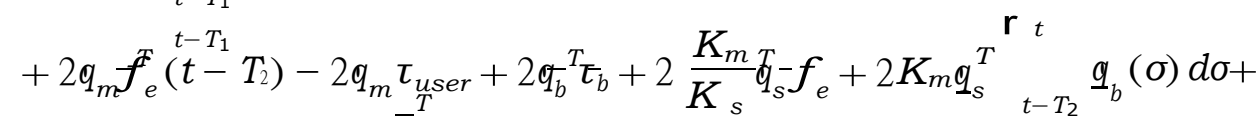

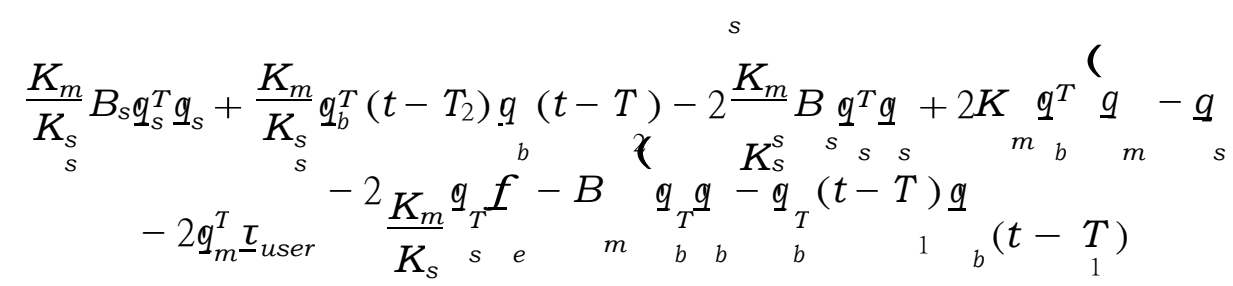

$$
\begin{aligned}
& +\frac{K_{m}}{K_{s}} B_{s}\left(\mathfrak{q}_{b}^{T} \underline{q}_{b}-\mathfrak{q}_{b}^{T}\left(t-T_{2}\right) \underline{q}_{b}\left(t-T_{2}\right)+\bar{T}_{1} \mathfrak{q}_{b}^{T} S \underline{b}\right. \\
& -{ }_{t-T_{1}}^{r^{s}} q_{b}^{T}(\sigma) S q_{b}(\sigma) d \sigma+\bar{T}_{2} q_{b}^{T} Z q_{b}-{ }_{t-T_{2}}^{r} \bar{q}_{b}^{T}(\sigma) Z q_{b}(\sigma) d \sigma
\end{aligned}
$$

(A6) can be simplified and rearranged as follows:

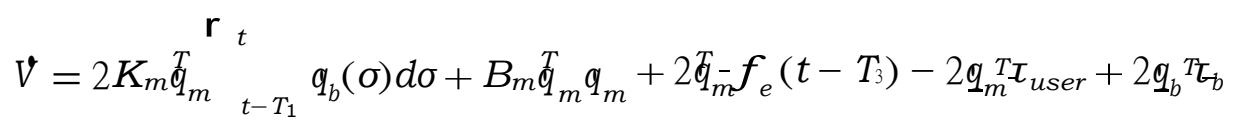

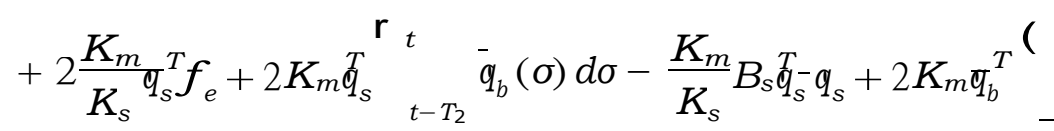

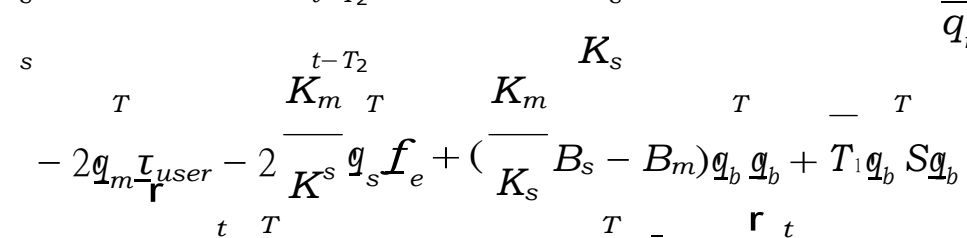

$$
\begin{aligned}
& -{ }_{t-T_{1}}^{t} \boldsymbol{q}_{b}^{T}(\sigma) S q_{b}(\sigma) d \sigma+\bar{T}_{2} q_{b} \bar{Z}_{q_{b}}-{ }_{t-T_{2}}^{r} \bar{q}_{b}^{T}(\sigma) Z q_{b}(\sigma) d \sigma
\end{aligned}
$$

Following inequalities are based on the Lemma 1:

$$
\begin{aligned}
& 2 K_{m} q_{m}^{-{ }_{m}^{T}{ }_{t-T_{1}}^{r_{t}}} q_{b}(\sigma) d \sigma-{ }_{t-T_{1}}^{\mathbf{r}_{t}} q_{b}^{T}(\sigma) S \underline{q}_{b}(\sigma) d \sigma \leq \bar{T}_{1} K_{m}{ }^{2} q_{m}^{T} S^{-1} q_{m} \\
& t-T_{1} \quad t-T_{1}
\end{aligned}
$$

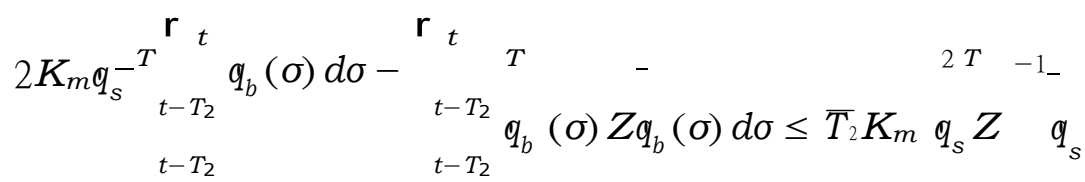

Substituting (A8) and (A9) into (A7) and rearranging:

$$
\begin{aligned}
& V=q_{m}^{T}{ }_{T} T_{1} K_{m}^{2} S^{-1}+B_{m} q_{m}+q_{b}^{T} \stackrel{(}{T_{1} S+T_{2}} Z+\frac{K_{m}}{K_{s}} B_{s}-B_{m} q_{b} \\
& +q^{T} \quad T K^{2} Z^{-1}-\frac{K_{m}}{B} \quad \underline{q}+2 q^{T}\left(f \quad K \quad \begin{array}{lll}
K_{s} & (t-T)-2 \underline{\tau} & )+2 q^{T}(K
\end{array}\right.
\end{aligned}
$$


$-\underline{q}+\underline{\tau})$

user

$m \quad m$

A 10$)$ 
With the conditions given by (15) for the last two terms of the (A10), and with the following inequalities being satisfied:

$$
\begin{gathered}
\bar{T}_{1} K_{m}{ }^{2} S^{-1}+B_{m} \leq 0 \\
T_{1} S+\bar{T}_{2} Z+\frac{K_{m}}{K_{s}} B_{s}-B_{m} \leq 0 \\
\bar{T}_{2} K_{m}{ }^{2} Z^{-1}-\frac{K_{m}}{K_{s}} B_{s} \leq 0
\end{gathered}
$$

the expression (A10) shows the negative semi-definiteness of $V$, implying the boundedness of $q_{i}$ and $q_{m}-q_{s}$ and hence the Lyapunov stability of the proposed bilateral haptic loop. 